\title{
Patients With First-Episode Psychosis are Not a Homogeneous Population: Implications for Treatment
}

\author{
Angelo Cocchi ${ }^{1, *}$, Giorgio Cerati ${ }^{2}$, Antonio Lora ${ }^{3}$, Anna Meneghelli ${ }^{1}$, Emiliano Monzani ${ }^{1}$, \\ Mauro Percudani ${ }^{4}$, Lorenzo Petrovich ${ }^{5}$, Fiorino Mirabella ${ }^{6}$, Angelo Picardi ${ }^{6}$ and Antonio Preti ${ }^{1,7}$
}

\begin{abstract}
${ }^{I}$ Dipartimento di Salute Mentale: Programma2000, Azienda Ospedaliera Ospedale Niguarda Ca' Granda, Milan, Italy; ${ }^{2}$ Dipartimento di Salute Mentale, Azienda Ospedaliera Ospedale Civile di Legnano, Italy; ${ }^{3}$ Dipartimento Salute Mentale, Azienda Ospedaliera della Provincia di Lecco, Italy; ${ }^{4}$ Dipartimento di Salute Mentale - A.O. "G. Salvini", Garbagnate Milanese, Italy; ${ }^{5}$ Comitato Tecnico per le Innovazioni in Salute Mentale. Direzione Generale Sanità - Regione Lombardia, Milan, Italy; ${ }^{6}$ Reparto Salute Mentale, Centro Nazionale di Epidemiologia Sorveglianza e Promozione della Salute, Istituto Superiore di Sanità, Rome, Italy; ${ }^{7}$ Dipartimento di Psicologia, Università di Cagliari, Italy
\end{abstract}

\begin{abstract}
Objective: This study aimed at defining the characteristics of a population of patients diagnosed with firstepisode psychosis (FEP), and accessing for the first time a center for early intervention in psychosis in the health district of Milan and its surroundings. Methods: Patients were included in the study from January 2007 to December 2008; criteria: first contact with any public mental health service of the catchment area for a first episode of schizophrenia or related syndromes according to the ICD-10 criteria. Cluster analysis was used to divide patients into groups based on the main socio-demographic and clinical characteristics at presentation. Results: Overall, 91 FEP patients were enrolled in the study. Two clusters were identified, which differed principally by symptom profile. Patients in cluster $1(\mathrm{n}=36)$ had severe agitation, and a history of alcohol and/or substance abuse at presentation more often than those in cluster 2 ( $\mathrm{n}=55$ ), who were more likely to suffer at presentation from severe depression or apathy, anxiety, poor self-care, functional or work impairment and severe social withdrawal. After six months of treatment patients improved on almost all symptomatic dimensions on the Health of the Nation Outcome Scale and the Brief Psychiatric Rating Scale, with greater improvement in cluster 1 than in cluster 2. Conclusions: The findings of this study need replication in larger samples and on a wider severity scale. Nevertheless, the heterogeneity of patients with FEP might impact on treatment. Policymakers should recognize the importance of the diagnostic and outcome assessment in the treatment of severe mental disorders.
\end{abstract}

Keywords: First episode psychosis, schizophrenia, early intervention, duration of untreated psychosis.

\section{INTRODUCTION}

The focus on early intervention in psychosis has widened interest in the characteristics that define the population of patients with psychosis at its onset [1-3]. The main target of these studies was the impact of the duration of untreated illness (DUI) or of untreated psychosis (DUP) on outcome, with many studies indicating longer DUP as associated with poor outcome $[4,5]$. However, the extreme variability in symptom presentation and in features such as DUP length [6], or co-morbidity with substance abuse or dependence [7], reveals that people diagnosed with first-episode psychosis belong to different populations, with potentially different illness courses and, hence, different treatment needs $[8,9]$.

Italy has undergone a deep-reaching reorganization of the mental health-care system in the past thirty years [10]. The complete closing down of the obsolete mental health hospitals (with the exception of forensic mental health hospitals) was accompanied by the development of a dedicated system

\footnotetext{
*Address correspondence to this author at the A.O. Ospedale Niguarda $\mathrm{Ca}^{\prime}$ Granda, Programma 2000 - Via Livigno, 3, 20128 Milan, Italy;

Tel: +39 0264445554 - +39 02 66809801; Fax: +39 02 66809809;

E-mails: angelo.cocchi@ospedaleniguarda.it and

programma2000@ospedaleniguarda.it
}

of mental health departments $[11,12]$. These departments are intertwined with general hospitals (where are the operating psychiatric wards for acute treatment), and a network of community services covering all the requirements of child, adolescent and adult populations [13, 14]. This community mental health-care network operates within the framework of a quasi-market, mixed private-public system of health-care provision, assuring patients freedom of choice between public and private centers of psychiatric care $[12,15]$. All psychiatric services are free of charge to patients and their families, as the costs of assessment and treatment are covered by general taxation, although some fees are paid for psychotherapy. The threshold for access to these services is very low, so patients can book a visit even without a formal indication by their general practitioner. Therefore, Italy is a suitable ground to study the characteristics of patients diagnosed with first-episode psychosis (FEP), since many of them are likely to get in touch with a psychiatric service in the first years of their illness.

This study aimed at defining the characteristics of a population of FEP patients accessing for the first time a center for early intervention in psychosis in the health district of Milan and its surroundings. Cluster analysis was used to di- 
vide patients into groups based on their main sociodemographic and clinical characteristics at presentation.

\section{METHODS}

The study was carried out from January 2007 to December 2008. It involved four centers for the early detection and intervention in psychosis operating within the Department of Mental Health of the following Hospital Authorities: "Niguarda Ca' Granda" Hospital, in Milan; the Psychiatric Unit of Bollate, subordinate to the "G. Salvini" Hospital, in Garbagnate Milanese, a small town near Milan; the Civic Hospital of Legnano, another small town near Milan; the Civic Hospital of Desio and Vimercate, two suburban towns near Milan. Milan is the main town of Lombardy, the largest and most affluent Region in Italy.

The Mental Health Unit of the National Center of Epidemiology, Surveillance and Health Promotion of the Italian National Institute of Health (INIH) was the steering committee and supervised the study from a statistical and methodological viewpoint.

\section{Setting of the Study}

The Programma2000, operating in Milan since 1999 under the Health Authority of the Niguarda Ca' Granda Hospital, was the first center to be opened in Italy with the aim of providing early detection and intervention on people with psychosis at its onset $[16,17]$, and served as a pilot program for the development of other centers in the area.

The Programma2000 covers a catchment area catering for approximately 200,000 inhabitants.

The early intervention center of the Civic Hospital of Desio and Vimercate was established in 2006 and covers a catchment area of about 230,000 residents, north of Milan.

The early intervention center of the Civic Hospital of Legnano (Milan) was established in 2005 and covers a catchment area of about 370,000 residents.

The early intervention center of the Psychiatric Unit of Bollate of to the "G. Salvini" Hospital (Garbagnate Milanese) was established in 2006 and covers a catchment area of about 95,000 residents.

The four catchment areas together cater for 895,000 inhabitants.

Each center is lead by a senior psychiatrist and its staff includes: two or more psychiatrists, operating in the center on a rotational scheme; two or more licensed psychotherapists; two or more clinical psychologists; two or more educators or nurses; a variable number of psychologists and educators in training.

All centers provide prompt intervention (within 24 hours) to the referred patients, who are offered a comprehensive, multidimensional evaluation with a package of standardized assessment instruments aimed at evaluating general psychopathology, level of functioning and associated impairment, disability and cognitive deficits [details in 16, 17].

All centers operate on an outpatient basis; admissions, when necessary, are negotiated with the General Hospital Psychiatric Units (GHPUs) operating in the same area. The beds available for hospital treatment were 10 to 15 per 100,000 when the study was performed.

All interventions are free of charge for the enrolled patients and are financed through a special grant from the Lombardy Regional Authority. Further details on the organization of the mental health system operating in Lombardy were reported elsewhere [18].

\section{Assessment and Criteria for Enrollment}

The patients were assessed using the following standardized assessment instruments: (i) a socio-demographic form; (ii) the Early Recognition Inventory Retrospective Assessment of Symptoms checklist (ERIraos-CL), a 17-item screening checklist intended to select persons needing a more in-depth assessment $[19,20]$; (iii) the Health of the Nation Outcome Scale (HoNOS), to assess psychopathology and disability: it includes 12 five-point items to evaluate clinical and social functioning over the prior 2 weeks [21, 22]; (iv) the 24-item Brief Psychiatric Rating Scale (BPRS), to assess general psychopathology [23, 24]; (v) the Global Assessment of Functioning (GAF) [25]; (vi) and (vii) the World Health Organization Disability Assessment Schedule (WHODAS) version II [26].

Patients were included in the study if they were between 17 and 30 years of age, and had been referred to any of the four participating centers after a first contact with any public mental health service of the catchment area for a first episode of psychosis (i.e. they had never received antipsychotic treatment before the current episode). Referral sources were mental-health professionals and associated surgeries, family physicians, or direct family referrals in response to awareness campaigns; self-referral was also allowed.

The main criterion for inclusion was a diagnosis of schizophrenia or related syndromes (F20-29 in ICD-10) according to the ICD-10 criteria [27]. Affective psychosis (bipolar disorder, or unipolar disorder with psychotic features) was an exclusion criterion, as was a co-morbid persistent substance-use dependent disorder, while substance use/abuse without dependence was not. Data on substance use, abuse and dependence were based on a detailed interview with the patient and a key informant (a close relative, usually a parent). Substance abuse was investigated by asking whether the patient had received any diagnosis of substance abuse in the last 24 months, or habitually consumed a drug from a list including alcohol, tobacco, cannabis, cocaine, heroin/opiates, amphetamine and its derivatives, hallucinogens and a residual class of "others". The threshold for abuse was defined according to the ICD-10 criteria, i.e. the inability to stop using the drug despite awareness of a health risk [27].

The past diagnoses received by the patients in the 12 months preceding the enrollment were considered too. In Lombardy both public and private psychiatrists are expected to provide a diagnosis according to ICD-10 criteria to justify the prescription of a drug treatment [18].

Additional information was collected during the interview of the patient and the key informant. Duration of untreated illness (DUI) and DUP were both measured as the time elapsed from the onset of key symptoms (anxiety, depression or social withdrawal for DUI; hallucinations, delu- 
sions or bizarre behavior for DUP) to the beginning of treatment (pharmacotherapy or psychotherapy) prescribed by a psychiatrist; DUP was measured in days, DUI in months. To measure DUP/DUI, researchers considered the symptoms as they were elicited by the ERIraos-CL, and the patient's estimated time of onset of key symptoms as listed in the tool. A therapist (usually a psychiatrist) and a researcher (usually a psychologist or an educator) of the team made the DUI/DUP assessment jointly. In problematic cases consensus with a senior clinician was sought.

The enrolled patients received a comprehensive, tailored and flexible intervention package. Prescribed tailored interventions included individual psychoeducational and motivational sessions, cognitive-behavioral psychotherapy, individual family psychoeducation and support, therapeutic group activities (e.g., anxiety management, assertive and problem-solving training, substance abuse prevention, etc.), various social group activities (e.g., music, multimedia, empowerment, computer training sessions, language classes, etc.), and supportive interventions on employment, school, compliance with medication, and planning of recreational activities [16, 17].

The institutional review board of the participating centers and of the INIH approved the protocol of the study, which conforms to the provisions of the 1995 Declaration of Helsinki (as revised in Tokyo, 2004). All patients gave their informed consent.

\section{Statistical Analyses}

All analyses were carried out with the Statistical Package for Social Science version 17.0. All analyses were twotailed, and statistical threshold was set at $\mathrm{p}<0.05$.

Cluster analysis was used to group subjects into clusters identified by typological characteristics that were not defined a-priori, were homogeneous within each cluster and heterogeneous between clusters. A two-step method was used to deal with both continuous and categorical variables and to determine the optimal number of clusters automatically. Likelihood ratio was used to determine the minimum distance between clusters across variables, in order to minimize within-cluster variation and maximize between-cluster variation. Model selection of grouping was based on the Bayesian Information Criterion (BIC) [28]. Chi-square for categorical variables, and the Student t-test for continuous variables were used to compare clusters on the variables that defined the model.

Multi-dimensional scales were entered in the cluster analysis after their reduction to the main factors. Since there is no consistent factor structure for both the BPRS and the HoNOS [29-32], principal component analysis with Varimax rotation was carried out de novo on both the BPRS and the HoNOS items (separately). The number of factors to be extracted was determined according to the scree-plot method [33]. Factor scores were calculated by summing up the score values of the items that saturated the factor. Six factors were extracted for the BPRS that explain $64 \%$ of total variance; and four factors were extracted for the HoNOS, explaining $60 \%$ of total variance (see Table 1 for details; solution available upon demand).
The following variables were entered to define the clusters: sex; age; education; severe agitation in current episode; severe depression or apathy in current episode; severe distress, anxiety or phobia in current episode; alcohol abuse in current episode; substance use in current episode; severe poor self-care in current episode; functional, social or work impairment in current episode; severe social withdrawal in current episode; HoNOS Behavioral problems, and HoNOS Mood and physical disability problems. All the other variables listed in Table 1 were used to further characterize the clusters once they had been defined.

\section{RESULTS}

The enrollment period lasted 12 months, at the end of which 91 FEP patients were enrolled in the study (Table 1).

Males $(n=71)$ prevailed on females $(n=20)$. Mean age in the sample was $22.5(S D=4.2)$. Most patients were unmarried and almost all had completed junior high school. Most were unemployed or declared to be students or housewives.

Anxiety disorders were an oft-reported diagnosis in the twelve months preceding the contact with the centers for the early detection and intervention on psychosis.

Cluster analysis identified two clusters, which differed principally by symptom level.

Patients included in cluster 2 were younger and more often unemployed than patients in cluster 1 . More often than those in cluster 1, cluster-2 patients were in treatment before they contacted the early intervention center, but less often had a hospital admission in their clinical history (see Table 1).

Patients in cluster 1 had severe agitation, and a history of alcohol and/or substance abuse at presentation more often than those in cluster 2, who were more likely, instead, to suffer from severe depression or apathy, anxiety, poor selfcare, functional or work impairment and severe social withdrawal at presentation.

Cluster-2 patients also had a younger age at first presentation than those in cluster 1, and a longer DUP. Detailed investigation of symptoms profile on the HoNOS, the BPRS and the WHODAS confirmed the differences observed at symptom presentation. Cluster-1 patients had more behavioral problems than those in cluster 2, who had more mood and physical disability problems. Levels of behavioral disorganization on the BPRS were higher in cluster 1, while impairment in the cognition and mobility dimensions was higher in cluster 2 .

\section{Six-Month Follow-Up}

Patients were reassessed after six months of treatment. There were no dropouts in the first six months of treatment.

Patients improved on almost all symptomatic dimensions on the HoNOS and the BPRS, with greater improvement in cluster 1 than in cluster 2 (Table 2).

The differences were more evident for the HoNOS factors concerning "Cognitive and social functioning problems" and "Mood and physical disability problems". However, the 
Table 1. General characteristics of the sample and results of cluster analysis.

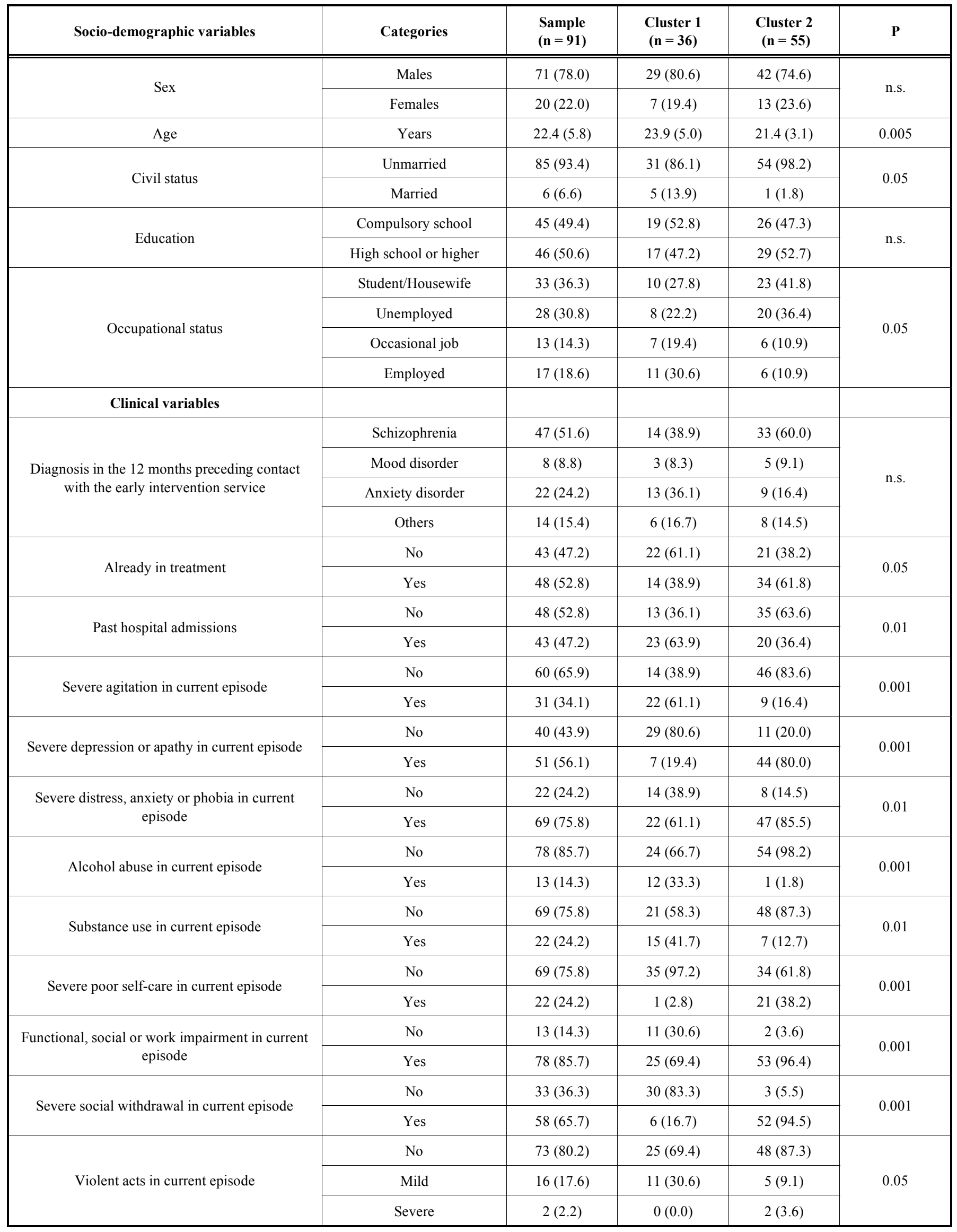


(Table 1) contd....

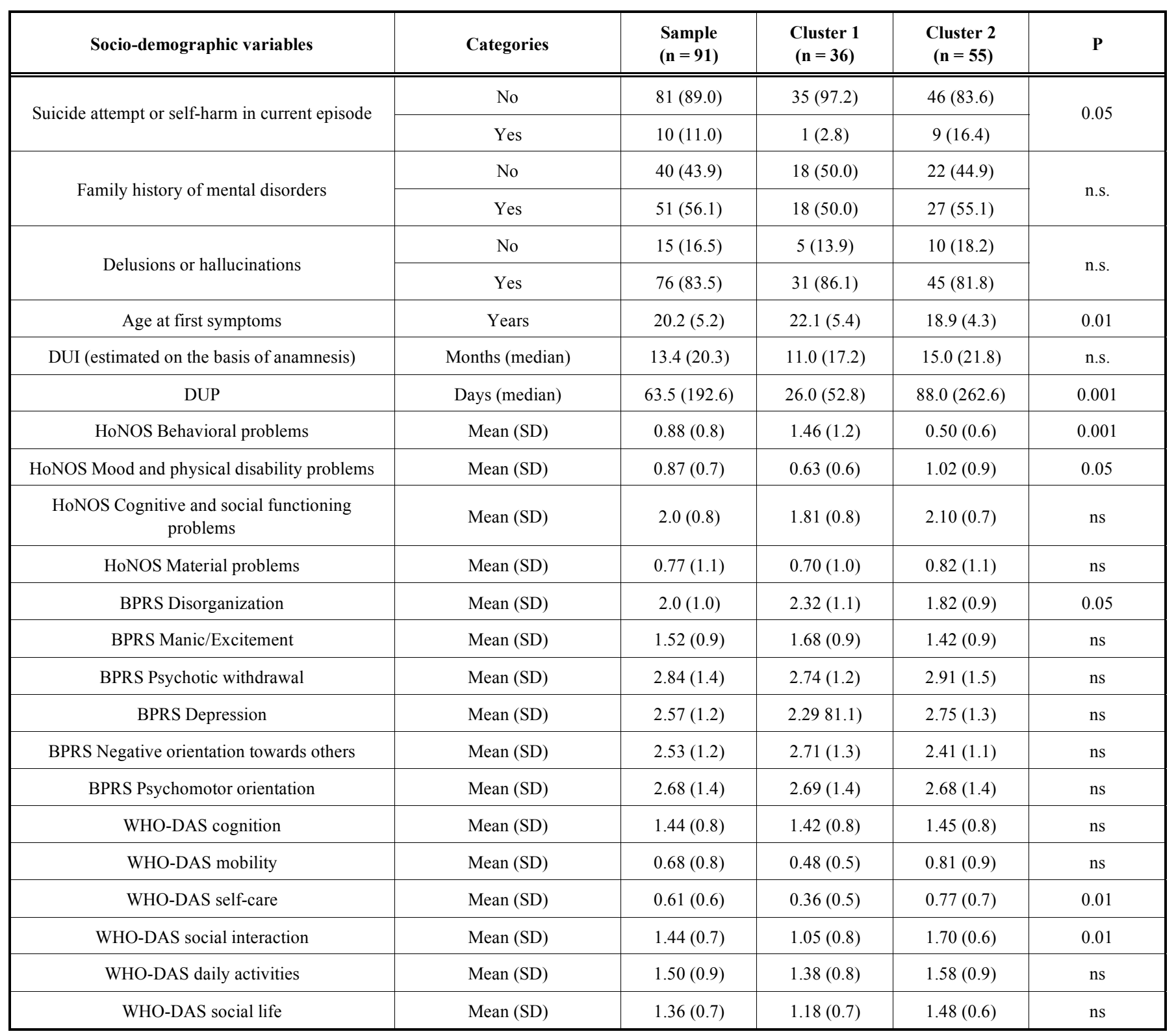

greatest difference between clusters concerned the BPRS factor "Negative orientation towards others", which decreased in cluster 1 but remained unchanged in cluster 2 .

\section{DISCUSSION}

The main findings of the study were the clear definition of two clusters of patients diagnosed with FEP among those accessing four centers for early intervention in psychosis in the health district of Milan and its surroundings; and the effectiveness of the therapeutic program on practically all the symptomatic dimensions assessed by the BRPS and the HoNOS.

Cluster-1 patients were older than those in cluster 2, and were characterized by behavioral symptoms such as aggressiveness, hyperactivity, agitation, alcohol and substance abuse, and poor self-care. Cluster-2 patients were characterized by symptoms of depression, apathy and social withdrawal. Worthy of note is the frequent occurrence of a diag- nosis of anxiety disorder in the twelve months preceding the contact with the early intervention center, a confirmation of the role of anxiety and of social anxiety, in particular [34], in the early phases of an incipient psychosis. Over a six-month follow-up interval, patients in cluster 1 were more ready to benefit from treatment than those in cluster 2, possibly because behavioral symptoms are more responsive to pharmacotherapy than negative symptoms such as apathy and social withdrawal.

The study findings point to the heterogeneity of patients with FEP. This heterogeneity may be attributed partially to the existence of two main types of psychosis, with different courses and outcomes: schizophrenia-like syndromes and affective psychoses $[35,36]$. The diagnosis of affective psychosis was an exclusion criterion at enrollment, but it is not always easy to distinguish a first episode within the schizophrenia spectrum from a first episode of mania or major depression with psychotic features. However, it is unlikely that 
Table 2. Six-month follow-up of 91 patients diagnosed with first-episode psychosis at four early intervention centers in Northern Italy. Mean and standard deviation at baseline and after six months of treatment.

\begin{tabular}{|c|c|c|c|c|}
\hline Variables & \multicolumn{2}{|c|}{ Cluster 1} & \multicolumn{2}{|c|}{ Cluster 2} \\
\hline HoNOS Mood and physical disability problems & $0.62(0.5)$ & $0.59(0.6)$ & $0.93(0.8)$ & $0.65(0.5)$ \\
\hline HoNOS Cognitive and social functioning problems & $1.82(0.7)$ & $1.07(0.7)$ & $2.23(0.7)$ & $1.60(0.7)^{\mathrm{a}, \mathrm{b}}$ \\
\hline BPRS Disorganization & $2.29(1.0)$ & $1.69(0.6)$ & $1.93(1.0)$ & $1.88(0.7)^{\mathrm{a}}$ \\
\hline BPRS Manic/Excitement & $1.61(0.7)$ & $1.50(0.5)$ & $1.51(1.1)$ & $1.40(0.4)$ \\
\hline BPRS Psychotic withdrawal & $2.64(1.2)$ & $1.7(1.0)$ & $3.11(1.4)$ & $2.07(0.8)^{\mathrm{a}}$ \\
\hline BPRS Depression & $2.19(0.9)$ & $1.86(0.8)$ & $2.63(1.1)$ & $2.23(0.9)^{\mathrm{a}}$ \\
\hline
\end{tabular}

Superscript legend:

$\mathrm{a}=\mathrm{p}<.01$ or lower in the comparison over time, from intake to 6-month follow up

$\mathrm{b}=\mathrm{p}<.01$ or lower in the comparison between groups

$\mathrm{c}=\mathrm{p}<.01$ or lower in the comparison groups by time

the differentiation of the sample into two clusters was merely the effect of the sparse cases of affective psychosis that went unrecognized at initial assessment. The two clusters resemble more the positive and negative syndromes described by Strauss et al. [37] and by Crow [38]. These two main syndromes were supposed to underlie different pathological mechanisms, with different responses to treatment and outcome. Subsequent studies provided evidence that a substantial proportion of patients were not stable over time and could display both types of symptoms $[39,40]$. Nevertheless, the distinction between positive and negative symptoms received some support within a dimensional model [41, 42].

Heterogeneity of patients with FEP may have an impact on treatment and, indeed, patients with prevailing behavioral symptoms had greater improvement at six-month follow-up than those with prevailing negative symptoms or depression. All patients received the same protocol of treatment. Better response to behavioral symptoms than to negative symptoms is an often-reported finding in the literature on schizophrenia [43]. However, the early identification of a subgroup of patients with prevailing negative symptoms may prompt dedicated intervention to overcome the known resistance of these symptoms to antipsychotic therapy. Evidence on the effectiveness of treatment over six months cannot be taken as evidence of the effectiveness of the early intervention protocol of care, since no control group on the usual treatment was available.

The study showed that protocols of early intervention can be implemented in the national, public mental health network of care. All participating centers were able to enroll their quote of patients, arriving at an incidence rate that is close to the expected incidence of schizophrenia-related psychoses in Italy [44]. In all centers data were collected according to the a-priori defined protocol of assessment without leakage or missing cases. Finally, all patients were retained in treatment according to the protocol for the whole duration of the study. These findings are promising in the perspective of spreading these programs over the country, as suggested by modern guidelines [45].

\section{Limitations of the Study}

Some limitations of the study must be acknowledged. This study uses a large array of variables to define patient status. As a matter of fact, with so many variables sample size might have prevented the definition of more finegrounded differences among clusters. Follow-up was short, too, and this might have limited inference on the long-term outcomes of the two identified clusters of FEP patients. Nevertheless, the findings of the study point to the importance of taking into account the variability of symptoms presentation in FEP patients and its impact on short-term outcome.

The setting, beside the limited sample size and the short follow-up, has influence on the generalizability of the findings. The sample was enrolled in four early intervention centers that operate on an outpatient basis and exclude patients with co-morbid persistent substance-use dependent disorder. The findings can be generalized to a subgroup of all FEP patients accessing psychiatric services in Italy.

\section{Implications for Research}

The findings of this study need replication in larger samples and with a more extended level of severity. The early detection and treatment of cases with psychosis in need of care is expected to reduce morbidity and its related disability, and to improve the long-term outcome [46, 47]. Indeed, early intervention protocols were found effective on the short term and able to sensibly reduce both direct and indirect costs of care as a reflection of their effectiveness [48-50]. The evidence on the medium-term effectiveness of these 
programs is unconvincing [51-53], and their costeffectiveness has been questioned [54-56]. Therefore, further studies are necessary to determine whether "heterogeneitysensitive" services are actually more effective than "generalist" services.

\section{Implications for Clinicians or Policymakers}

Whatever the real effectiveness of third-tier services dedicated to the early detection and treatment of psychosis, there remains the need of identifying different profiles of symptoms presentation in FEP patients, since these different profiles can impact on short- and long-term outcomes. Albeit the findings of this study are in need of replication, we think that the two clusters we identified must be distinguished in clinical settings. To this aim, a detailed assessment is necessary to gain insight of the profile of patient's symptoms with attention being paid to both positive and negative symptoms. Negative symptoms such as anhedonia, apathy, autism and avolition are often overlooked in the Italian mental health system of care, with greater attention being devoted to the diagnosis and treatment of the symptoms that are more disturbing to the community: agitation and the disordered behaviors triggered by delusions and hallucinations.

Policymakers should recognize the importance of assessment in the treatment of severe mental disorders and fund appropriate protocols to diffuse the practice of assessing clinical status at enrollment and applying state-of-the-art outcome measures to the follow-up.

\section{CONCLUSIONS}

In Italy about a half of the patients diagnosed with schizophrenia does not receive the minimum adequate treatment, particularly those at their first episode [57]. Consideration of the heterogeneity of patients with FEP might improve care. Indeed, a detailed assessment at intake can allow the prescription of the best, tailored treatment. Improved allocation of the resources available to treatment may also cut costs [58]. This is a clear advantage in an era of financial crisis and saving on costs.

\section{CONFLICT OF INTEREST}

The author(s) confirm that this article content has no conflicts of interest.

\section{ACKNOWLEDGEMENT}

The study was financed by a Grant of the Ministero della Salute, Programma di Ricerca Finalizzata D.lgs 502/92 ex art. 12 - Bando 2005 and by Regione Lombardia, Decreto DGS n. 9676 del 4.9.2006.

This paper is dedicated to the memory of Pierluigi Morosini, M.D., former Head of the Mental Health Unit of the National Institute of Health, who enthusiastically supported and contributed to the development and conduct of this research project.

\section{REFERENCES}

[1] McGorry PD, Killackey E, Yung AR. Early intervention in psychotic disorders: detection and treatment of the first episode and the critical early stages. Med J Aust 2007; 187(7 Suppl): S8-10.
[2] Kirkbride JB, Stubbins C, Jones PB. Psychosis incidence through the prism of early intervention services. Br J Psychiatry 2012; 200: 156-7.

[3] Rajapakse T, Garcia-Rosales A, Weerawardene S, Cotton S, Fraser $\mathrm{R}$. Themes of delusions and hallucinations in first-episode psychosis. Early Interv Psychiatry 2011; 5: 254-8.

[4] Marshall M, Lewis S, Lockwood A, Drake R, Jones P, Croudace T. Association between duration of untreated psychosis and in cohorts of first-episode outcome patients - a systematic review. Arch Gen Psychiatry 2005; 62: 975-83.

[5] Perkins DO, Gu H, Boteva K, Lieberman JA. Relationship between duration of untreated psychosis and outcome in first-episode schizophrenia: a critical review and meta-analysis. Am J Psychiatry 2005; 162: 1785-804.

[6] Singh S. Outcome measures in early psychosis. Relevance of duration of untreated psychosis. Br J Psychiatry 2007; 191(suppl. 50): S58-S63.

[7] Lambert M, Conus P, Lubman DI, et al. The impact of substance use disorders on clinical outcome in 643 patients with first-episode psychosis. Acta Psychiatr Scand 2005; 112: 141-8.

[8] Mura G, Petretto DR, Bhat KM, Carta MG. Schizophrenia: from epidemiology to rehabilitation. Clin Pract Epidemiol Ment Health 2012; 8: 52-66

[9] Colasanti A, Paletta S, Moliterno D, Mazzocchi A, Mauri MC, Altamura AC. Symptom dimensions as predictors of clinical outcome, duration of hospitalization, and aggressive behaviours in acutely hospitalized patients with psychotic exacerbation. Clin Pract Epidemiol Ment Health 2010; 6: 72-8.

[10] Lora A. An overview of the mental health system in Italy. Ann Ist Super Sanita 2009; 45(1): 5-16.

[11] de Girolamo G, Cozza M. The Italian psychiatric reform: a 20-year perspective. Int J Law Psychiatry 2000; 23: 197-214.

[12] Fattore G, Percudani M, Pugnoli C, Contini A, Beecham J. Mental health care in Italy: organisational structure, routine clinical activity and costs of a Community Psychiatric Service in Lombardy Region. Int J Soc Psychiatry 2000; 46: 250-65.

[13] de Girolamo G, Barbato A, Bracco R, et al. The characteristics and activities of acute psychiatric inpatient facilities: A national survey in Italy. Br J Psychiatry 2007; 191: 170-7.

[14] Gigantesco A, Miglio R, Santone G, et al. The process of care in general hospital psychiatric units: a national survey in Italy. Aust NZ J Psychiatry 2007; 41: 509-18.

[15] Preti A, Rucci P, Santone G, et al. Patterns of admission to acute psychiatric inpatient facilities: A national survey in Italy. Psychol Med 2009; 39: 485-96.

[16] Cocchi A, Meneghelli A, Preti A. Programma 2000: Celebrating ten years of activity of an Italian pilot program on early intervention in psychosis. Aust NZ J Psychiatry 2008; 42: 1003-12.

[17] Meneghelli A, Cocchi A, Preti A. Programma2000: A multi-modal pilot program on early intervention in psychosis underway in Italy since 1999. Early Interv Psychiatry 2010; 4: 97-103.

[18] Lora A, Barbato A, Cerati G, Erlicher A, Percudani M. The mental health system in Lombardy, Italy: access to services and pattern of care. Soc Psychiatry Psychiatr Epidemiol 2012; 47: 447-54.

[19] Häfner H, Riecher-Rossler A, Hambrecht M. IRAOS: An instrument for the assessment of onset and early course of schizophrenia. Schizophr Res 1992; 6: 209-23.

[20] Raballo A, Meneghelli A, Cocchi A, et al. Shades of vulnerability: latent structures of clinical caseness in prodromal and early phases of schizophrenia. Eur Arch Psychiatry Clin Neurosci 2013; [Epub ahead of print]

[21] Wing JK, Beevor AS, Curtis RH, Park BG, Hadden S, Burns A. Health of the Nation Outcome Scales (HoNOS): research and development. Br J Psychiatry 1998; 172: 11-8.

[22] Preti A, Pisano A, Cascio MT, et al. Validation of the Health of the Nation Outcome Scales as a routine measure of outcome in early intervention programs. Early Interv Psychiatry 2012; 6: 423-31.

[23] Overall JE, Gorham DE. The brief psychiatric rating scale. Psychol Rep 1962; 10: 799-812.

[24] Roncone R, Ventura J, Impallomeni M, et al. Reliability of an Italian standardized and expanded Brief Psychiatric Rating Scale (BPRS 4.0) in raters with high vs. low clinical experience. Acta Psychiatr Scand 1999; 100: 229-36.

[25] Moos RH, McCoy L, Moos BS. Global assessment of functioning (GAF) ratings: determinants and roles as predictors of one-year treatment outcomes. J Clin Psychol 2000; 56: 449-61. 
[26] Federici S, Meloni F, Mancini A, Lauriola M, Olivetti Belardinelli M. World Health Organisation Disability Assessment Schedule II: contribution to the Italian validation. Disabil Rehabil 2009; 31: 553-64.

[27] WHO (World Health Organization). Tenth Revision of the International Classification of Diseases and Related Health Problems (ICD-10). Geneve: WHO Press 1992

[28] Schwarz G. Estimating the dimension of a model. Ann Stat 1978; 6: 461-4.

[29] Shafer A. Meta-analysis of the brief psychiatric rating scale factor structure. Psychol Assess 2005; 17: 324-35.

[30] Picardi A, Viroli C, Tarsitani L, et al. Heterogeneity and symptom structure of schizophrenia. Psychiatry Res 2012; 198: 386-94.

[31] Trauer T. The subscale structure of the Health of the Nation Outcome Scales (HoNOS). J Ment Health 1999; 8: 499-509.

[32] Andreas S, Harfst T, Rabung S, et al. The validity of the german version of the health of the nation outcome scales (HoNOS-D): a clinician-rating for the differential assessment of the severity of mental disorders. Int J Methods Psychiatr Res 2010; 19: 50-62.

[33] Cattell RB. The scree test for the number of factors. Multivar Behav Res 1966; 1: 245-76.

[34] Michail M, Birchwood M. Social anxiety disorder in first-episode psychosis: incidence, phenomenology and relationship with paranoia. Br J Psychiatry 2009; 195: 234-41.

[35] Whitty P, Clarke M, McTigue O, et al. Diagnostic stability four years after a first episode of psychosis. Psychiatr Serv 2005; 56 : 1084-8.

[36] Kim JS, Baek JH, Choi JS, Lee D, Kwon JS, Hong KS. Diagnostic stability of first-episode psychosis and predictors of diagnostic shift from non-affective psychosis to bipolar disorder: a retrospective evaluation after recurrence. Psychiatry Res 2011; 188: 29-33.

[37] Strauss JS, Carpenter WT, Bartko J. The diagnosis and understanding of schizophrenia. III. Speculations on the processes that underlie schizophrenic symptoms and signs. Schizophr Bull 1974; 1: 619.

[38] Crow TJ. Molecular pathology of schizophrenia: more than a disease process? Br Med J 1980; 280: 66-8.

[39] Marneros A, Diester A, Rohde A. Long-term investigation in stability of positive/negative distinction. In Marneros A, Andreasen NC, Tsuang M Eds. Negative vs Positive Schizophrenia. Berlin: Springer 1991.

[40] Peralta V, de Leon J, Cuesta MJ. Are there more than two syndromes in schizophrenia? A critique of the positive-negative dichotomy. Br J Psychiatry 1992; 161: 335-43.

[41] Fenton WS, McGlashan TH. Natural history of schizophrenia subtypes. II. Positive and negative symptoms and long-term course. Arch Gen Psychiatry 1991; 48: 978-86.

[42] PeraltaV, Cuesta MJ, de Leon J. Positive and negative symptoms/syndromes in schizophrenia: reliability and validity of different diagnostic systems. Psychol Med 1995; 25: 43-50.
[43] Lang FU, Kösters M, Lang S, Becker T, Jäger M. Psychopathological long-term outcome of schizophrenia -- a review. Acta Psychiatr Scand 2013; 127: 173-82.

[44] Gigantesco A, Lega I, Picardi A; SEME Collaborative Group. The Italian SEME Surveillance System of Severe Mental Disorders Presenting to Community Mental Health Services. Clin Pract Epidemiol Ment Health 2012; 8: 7-11.

[45] De Masi S, Sampaolo L, Mele A, et al. The Italian guidelines for early intervention in schizophrenia: Development and Conclusions. Early Interv Psychiatry 2008; 2: 291-302.

[46] McGorry PD. Preventive strategies in early psychosis: verging on reality. Br J Psychiatry 1998; 172(suppl 33): 1-2.

[47] Edwards J, McGorry PD. Implementing early intervention in psychosis. A guide to establishing early psychosis services. London, UK: Dunitz 2002.

[48] McCrone P, Craig TK, Power P, Garety PA. Cost-effectiveness of an early intervention service for people with psychosis. Br J Psychiatry $2010 ; 196: 377-82$.

[49] Cocchi A, Mapelli V, Meneghelli A, Preti A. Cost-effectiveness of treating first episode psychosis. Five-year follow-up results from an Italian early intervention program. Early Interv Psychiatry 2011; 5: 203-11.

[50] Hastrup LH, Kronborg C, Bertelsen M, et al. Cost-effectiveness of early intervention in first-episode psychosis: economic evaluation of a randomised controlled trial (the OPUS study). Br J Psychiatry 2013; 202: 35-41.

[51] Bertelsen M, Jeppesen P, Petersen L, et al. Five-year follow-up of a randomized multicenter trial of intensive early intervention vs standard treatment for patients with a first episode of psychotic illness. Arch Gen Psychiatry 2008; 65: 762-71.

[52] Gafoor R, Nitsch D, McCrone P, et al. Effect of early intervention on 5-year outcome in non-affective psychosis. Br J Psychiatry 2010; 196: 372-6.

[53] Marshall M, Rathbone J. Early intervention for psychosis. Cochrane Database Syst Rev 2011; 6: CD004718.

[54] Amos A. Assessing the cost of early intervention in psychosis: a systematic review. Aust N Z J Psychiatry 2012; 46: 719-34.

[55] Mihalopoulos C, McCrone P, Knapp M, Johannessen JO, Malla A, McGorry P. The costs of early intervention in psychosis: restoring the balance. Aust N Z J Psychiatry 2012; 46: 808-11.

[56] Valmaggia LR, McGuire PK, Fusar-Poli P, Howes O, McCrone P. Economic impact of early detection and early intervention of psychosis. Curr Pharm Des 2012; 18: 592-5.

[57] Lora A, Conti V, Leoni O, Rivolta AL. Adequacy of treatment for patients with schizophrenia spectrum disorders and affective disorders in Lombardy, Italy. Psychiatr Serv 2011; 62: 1079-84.

[58] Cocchi A, Mapelli V, Meneghelli A, Preti A. Cost-effectiveness of treating first episode psychosis. Five-year follow-up results from an Italian early intervention program. Early Interv Psychiatry 2011; 5: 203-11.

\begin{tabular}{lrr}
\hline Received: October 19, 2013 & Revised: January 20, 2014 & Accepted: January 22, 2014 \\
(C) Cocchi et al.; Licensee Bentham Open. &
\end{tabular}

(C) Cocchi et al.; Licensee Bentham Open.

This is an open access article licensed under the terms of the Creative Commons Attribution Non-Commercial License (http://creativecommons.org/licenses/by-nc/3.0/) which permits unrestricted, non-commercial use, distribution and reproduction in any medium, provided the work is properly cited. 